\title{
A DNS study of aerosol and small-scale cloud turbulence interaction
}

\author{
Natalia Babkovskaia ${ }^{1}$, Ullar Rannik ${ }^{1}$, Vaughan Phillips ${ }^{2}$, Holger Siebert $^{3}$, Birgit Wehner $^{3}$, and Michael Boy ${ }^{1}$ \\ ${ }^{1}$ University of Helsinki, Department of Physics, Helsinki, Finland \\ ${ }^{2}$ Lund University, Department of Physical Geography and Ecosystems Science, Lund, Sweden \\ ${ }^{3}$ Leibniz Institute for Tropospheric Research, Leipzig, Germany
}

Correspondence to: Natalia Babkovskaia (nbabkovskaia@gmail.com)

Received: 9 November 2015 - Published in Atmos. Chem. Phys. Discuss.: 22 January 2016

Revised: 20 May 2016 - Accepted: 1 June 2016 - Published: 29 June 2016

\begin{abstract}
The purpose of this study is to investigate the interaction between small-scale turbulence and aerosol and cloud microphysical properties using direct numerical simulations (DNS). We consider the domain located at the height of about $2000 \mathrm{~m}$ from the sea level, experiencing transient high supersaturation due to atmospheric fluctuations of temperature and humidity. To study the effect of total number of particles $\left(N_{\text {tot }}\right)$ on air temperature, activation and supersaturation, we vary $N_{\text {tot }}$. To investigate the effect of aerosol dynamics on small-scale turbulence and vertical air motion, we vary the intensity of turbulent fluctuations and the buoyant force. We find that even a small number of aerosol particles $\left(55.5 \mathrm{~cm}^{-3}\right)$, and therefore a small droplet number concentration, strongly affects the air temperature due to release of latent heat. The system comes to an equilibrium faster and the relative number of activated particles appears to be smaller for larger $N_{\text {tot. }}$. We conclude that aerosol particles strongly affect the air motion. In a case of updraught coursed by buoyant force, the presence of aerosol particles results in acceleration of air motion in vertical direction and increase of turbulent fluctuations.
\end{abstract}

\section{Introduction}

Interaction of atmospheric turbulence with aerosol and cloud formation processes has been studied extensively. Due to non-linearity of particle formation and other aerosol dynamical processes, the fluctuations of temperature and relative humidity can have strong effect on aerosol formation. Largescale fluctuations of atmospheric properties, which occur for example in the atmospheric boundary layer, can drive the initiation of particle formation (Easter et al., 1994). A mixing of air with different properties, including temperature and relative humidity, has been shown to enhance atmospheric nucleation significantly (Nilsson and Kulmala, 1998). More specifically, the atmospheric waves can increase the nucleation rate by several orders of magnitude and also affect the size spectrum of the particles (Nilsson et al., 2000). Furthermore, the activation of atmospheric particles in cloud areas is affected by the fluctuation of supersaturation. Some droplets were shown to also grow in undersaturated conditions due to fluctuations and the bimodal particle size distribution could be observed after the initially unimodal particle population experienced fluctuating supersaturation (Kulmala et al., 1997).

In-cloud turbulence has been shown to intensify cloudmicrophysical processes, determining cloud properties (Benmoshe and Khain, 2014). Similarly, aerosol loadings also influence the numbers and sizes of cloud particles, thereby influencing precipitation, cloud extent and the climate (Forster, 2007). Both turbulence and aerosol particles can influence the chemical reactions in the atmosphere, providing surfaces for aqueous phase chemistry and promoting uptake of gaseous species. This can have repercussions for chemical reactions in the air. Yet both environmental factors, namely aerosol composition and turbulence, are usually considered to be separate influences.

The large-scale atmospheric turbulence is well known to affect aerosol processes significantly. In turn, aerosol properties may influence the buoyancy and turbulence. A motivation for a potential aerosol-turbulence interaction is that the most advanced cloud-microphysical models can now resolve the cloud edges where mixing occurs and where spatial gradients are great in turbulence and concentrations of hydrometeors, including aerosols. Consequently, by including 
a more complete set of such interactions in the models, the simulation of aerosols, turbulence and microphysics may be improved.

The main goal of this direct numerical simulation (DNS) study is to investigate the interaction between small-scale turbulence and aerosol and cloud microphysical properties. The chosen DNS domain is realistic for a small volume at the cloud edge, where turbulent mixing is a dominate feature (Katzwinkel et al., 2014) and can create fluctuations in temperature as well as humidity. Such conditions, however, appear transient because the presence of atmospheric aerosols leads to a depletion of supersaturation via condensation and droplet activation.

We use a high-order public-domain finite-difference code, Pencil Code, for compressible hydrodynamic flows. The code is highly modular and comes with a large selection of physics modules. It is widely documented in the literature (Dobler et al., 2006; Pencil Code, 2001, and references therein). The chemistry module is responsible for the detailed description of the necessary quantities in a case of complicated chemical composition, such as diffusion coefficients, thermal conductivity, reaction rates etc. (Babkovskaia et al., 2011). A detailed description of the aerosol module can be found in Babkovskaia et al. (2015). This paper is constructed as follows: Section 2 is devoted to the description of the model, results are presented in Sect. 3, and Sect. 4 provides a summary of our study.

\section{Description of the model}

\subsection{Aerosol}

As a starting point for our simulations, we consider values typical for observations made in trade wind cumuli. During the CARRIBA (Cloud, Aerosol, Radiation and tuRbulence in the trade wInd regime over Barbados) project (Siebert et al., 2013, hereafter called SI13), total aerosol number concentrations in the marine sub-cloud layer up to a few $100 \mathrm{~cm}^{-3}$ were observed (Fig. 6f in SI13). Although it was argued that the highest values are due to local biomass burning, we consider a concentration of $550 \mathrm{~cm}^{-3}$ as typical and 55 and $5550 \mathrm{~cm}^{-3}$ as two extreme values for the sensitivity test. The initial normalized aerosol number size distribution, shown as a dotted line in Fig. 1, compares well with the shape of the observed distribution shown as red line in Fig. 8 of SI13.

We assume a soluble aerosol $(\mathrm{NaCl})$, which will dilute inside the droplet. We take 50 size bins, logarithmically distributed in the range $[10 \mathrm{~nm}, 1000 \mu \mathrm{m}]$. As an initial distribution of particles, we take the observational data at the sea level and assume that it is the same everywhere in the domain (see the distribution in Fig. 1). To analyse the effect of the total number of aerosol particles, $N_{\text {tot }}$, on the structure and properties of turbulent motion, we consider the following cases: $N_{\text {tot }}=55.5 \mathrm{~cm}^{-3}$ and initial supersaturation averaged
Table 1. The summary of key parameters for studying the effect of total number of particles on air temperature, supersaturation and activation. $S_{\mathrm{av}}$ is initial supersaturation averaged over the domain.

\begin{tabular}{lll}
\hline Case 1 & $N_{\text {tot }}=55.5 \mathrm{~cm}^{-3}$ & $S_{\mathrm{av}}=10.3 \%$ \\
Case 2 & $N_{\text {tot }}=555 \mathrm{~cm}^{-3}$ & $S_{\mathrm{av}}=10.3 \%$ \\
Case 3 & $N_{\text {tot }}=5550 \mathrm{~cm}^{-3}$ & $S_{\mathrm{av}}=10.3 \%$ \\
Case 4 & $N_{\text {tot }}=5550 \mathrm{~cm}^{-3}$ & $S_{\mathrm{av}}=0.6 \%$ \\
Case 5 & no particles & \\
\hline
\end{tabular}

over domain $S_{\mathrm{av}}=10.3 \%$ (case 1); $N_{\text {tot }}=555 \mathrm{~cm}^{-3}$ and $S_{\mathrm{av}}=10.3 \%$ (observed data, case 2); $N_{\mathrm{tot}}=5550 \mathrm{~cm}^{-3}$ and $S_{\mathrm{av}}=10.3 \%$ (case 3); $N_{\text {tot }}=5550 \mathrm{~cm}^{-3}$ and $S_{\mathrm{av}}=0.6 \%$ (case 4) and no particles (case 5) (see Table 1).

\subsection{Air composition}

We assume the following air composition $\mathrm{O}_{2}+\mathrm{H}_{2} \mathrm{O}+\mathrm{N}_{2}$, where nitrogen mass fraction is taken to be $Y_{\mathrm{N}_{2}}=70 \%$. The observations provide us with the absolute humidity to set the initial value for the mass fraction of water vapour, $Y_{\mathrm{H}_{2} \mathrm{O}}$. Oxygen mass fraction is recalculated from the normalization conditions; i.e. $Y_{\mathrm{O}_{2}}+Y_{\mathrm{H}_{2} \mathrm{O}}+Y_{\mathrm{N}_{2}}=1$, where $Y_{\mathrm{O}_{2}}$ is the oxygen mass fraction.

\subsection{Initial conditions}

This model represents the 3-D fluid flow on the microscale inside a volume of $10 \mathrm{~cm} \times 10 \mathrm{~cm} \times 10 \mathrm{~cm}$, just inside the cloud in the mid-troposphere. Based on data of CARRIBA observations typical for the upper parts of clouds/cloud edges at a height of $2000 \mathrm{~m}$, we set the initial conditions for air temperature $\left(T_{0}=285.4 \mathrm{~K}\right)$ and water vapour mixing ratio $\left(q_{0}=0.0124\right)$. The small vertical gradients of temperature and water content are also based on the CARRIBA measurements: the total differences between values of air temperature and water vapour mixing ratio at the upper and lower edges of the domain are $\Delta T=0.001 \mathrm{~K}$ and $\Delta q=4 \times 10^{-5}$, correspondingly.

Observed temperature and absolute humidity result in peak value of supersaturation $(S)$ of up to 0.1 (e.g. $10 \%$ ). Such values of $S$ are extremely high considering only an adiabatic lifting of a cloudy air parcel. This quasi-steady-state supersaturation depends mainly on vertical updraught velocity, droplet number concentration and mean droplet diameter, and appears to be on the order of a few tenths of percent for vertical updraughts of about $1 \mathrm{~m} \mathrm{~s}^{-1}$. However, it is well known that atmospheric clouds are mainly non-adiabatic due to turbulent mixing, and a few percent supersaturation is realistic for higher updraught velocities (e.g. Korolev and Mazin, 2003). This is particularly true at the cloud edges, where entrainment of unsaturated air into the cloud results in strong mixing and fluctuations of the water vapour and temperature field. It strongly depends on the correlation between these 


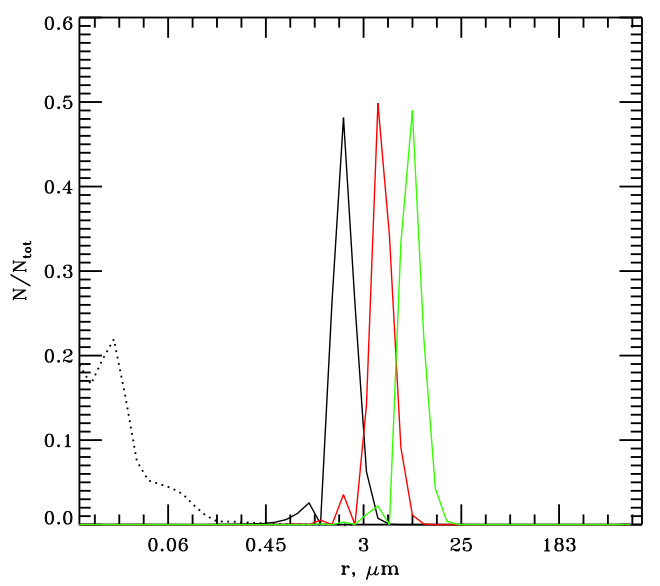

Figure 1. Relative distribution of particles averaged over the domain at $t=0 \mathrm{~s}$ (black dotted curve) and at $t=3 \mathrm{~s}$ (solid curve) for the cases of $N_{\text {tot }}=55.5 \mathrm{~cm}^{-3}$ (green solid curve), $N_{\text {tot }}=$ $555 \mathrm{~cm}^{-3}$ (red solid curve) and $N_{\text {tot }}=5550 \mathrm{~cm}^{-3}$ (black solid curve). Dotted curve corresponds to the observed distribution of aerosol; solid and dashed curves are distributions of droplets.

two thermodynamic fields whether strong fluctuations can result in high fluctuations of supersaturation or not. Until now we have not been aware of any observations of this correlation in clouds. Based on theoretical arguments and observations in the convective boundary layer below clouds, Kulmala et al. (1997) provided some convincing arguments that, at the cloud base, high fluctuations of supersaturation on the order of up to several percent can exist. It is also well known that turbulence in high Reynolds number flows (typical in convective clouds) is highly intermittent (Siebert et al., 2010). Shaw (2000) argued that under such conditions longliving vortex tubes could produce small areas with decreased droplet number concentrations and therefore high supersaturation, resulting in secondary activation. To summarize, there are good arguments that supersaturation fluctuations of a few percent can be generated without strong updraughts.

On the other hand, the supersaturation excess would be eliminated by condensation onto droplets, and quasisteady-state supersaturation would be restored (Korolev and Mazin, 2003). Therefore, the key question about the temporal timescale is under consideration now. The ratio of two timescales is important here: the phase relaxation time which describes how fast the supersaturation can react on the new thermodynamic condition and the turbulent mixing timescale which describes how fast turbulence can mix a certain volume (eddy with the length scale $l$ ). If the phase relaxation time is smaller than the turbulent mixing time then the actual supersaturation will tend to the quasi-steady-state solution. However, for scales where the turbulent mixing is faster we expect strong supersaturation fluctuations to "survive".

Let us now assume a small eddy of size $l=1 \mathrm{~m}$ and a local energy dissipation rate of $\epsilon=0.1 \mathrm{~m}^{2} \mathrm{~s}^{-3}$. These dissipation values are typical peak values for cumulus clouds on such small scales. The highest dissipation can be found at Kolmogorov size (see discussion in Siebert et al., 2006, 2010). The eddy turnover time is $\tau_{\text {eddy }}=\left(l^{2} / \epsilon\right)^{1 / 3} \approx 2 \mathrm{~s}$. If we now take a phase relaxation time of the order of $1 \mathrm{~s}$, which is typical for cumulus clouds (see again Korolev and Mazin, 2003), we see that these two timescales are of the same order. Therefore, we conclude that on scales below $1 \mathrm{~m}$, strong supersaturation fluctuations can exist and the quasi-steady-state solution should be considered as a mean value with superimposed fluctuations, with amplitudes up to several percent. This argumentation partly follows the discussion in Sect. 8e by Korolev and Mazin (2003).

Kulmala et al. (1997) estimated standard deviations of supersaturation of up to $5 \%$ based on aircraft observations at cloud level but outside the clouds. Ditas et al. (2012) observed supersaturation fluctuations in a field of stratocumulus clouds and estimated peak-to-peak values of up to $1.5 \%$ from highly collocated temperature and water vapour observations, which is much higher compared to the quasi-steadystate solution. It is straightforward to assume that higher $S$ values can be expected for parts of (shallow) cumulus clouds. Thus, we argue that for our small modelled volume a transient supersaturation of $10 \%$ is realistic for a specific mixing event.

Pressure, $p$, is assumed to be constant everywhere in the domain and it is also based on measurements. The air density, $\rho$, is calculated from the equation of state $p=\rho R T / m$, where $R$ is the gas universal constant and $m$ is the air molar mass. The initial velocity is taken to be zero.

To generate the initial turbulent field, we make the first 100 iterations without evaporation/activation of aerosol particles (further "aerosol dynamics"), including randomly directed external forces (see next section). After that the external forces are set to zero, whereas the particles start to evolve. Thus, the turbulence decays over the analysed time. The maximal time step allowed by the Courant condition for convergence is $\Delta t_{\mathrm{c}}=10^{-6} \mathrm{~s}$ (Courant time step). Since $\Delta t_{\mathrm{c}}$ is much larger than the time step needed to move the smallest particle to the neighbour size bin $\left(\Delta t_{\mathrm{a}}=2 \times 10^{-7} \mathrm{~s}\right)$, at every Courant time step we make five substeps and calculate the particle evolution equation only.

\subsection{Boundary conditions}

In all three directions we set periodic boundary conditions for all variables, including the number density function. It means that at every time step, $\Delta t_{\mathrm{c}}$, the number of particles appearing on the bottom/left boundaries is equal to the number of particles disappearing through the top/right boundaries. While the periodic boundary conditions modify the initial temperature stratification, they allow us to consider this domain as an isolated volume; i.e. the total mass, energy and number of particles in the domain does not change with time. In turn, it makes it possible to compare the results of simulations, 
Table 2. The summary of key parameters for studying the effect of aerosol on turbulence.

\begin{tabular}{lll}
\hline & Equilibrium & Non-equilibrium \\
\hline Low intensive & $T_{0}=285.4 \mathrm{~K}$ & $T_{0}=283.5 \mathrm{~K}$ \\
turbulence & $f_{0}=10$ & $f_{0}=10$ \\
\hline High & $T_{0}=285.4 \mathrm{~K}$ & $T_{0}=283.5 \mathrm{~K}$ \\
intensive turbulence & $f_{0}=100$ & $f_{0}=100$ \\
\hline
\end{tabular}

varying the key parameters of the model, and to carry out the detailed quantitative analysis of the interaction between aerosol and turbulence.

\subsection{Basic equations}

A detailed description of the main equations is presented by Babkovskaia et al. (2015). We consider the standard compressible Navier-Stokes system, including the equation for conservation of mass, momentum, energy and chemical species. The momentum of air is transported due to viscous force. To describe the gravitational effect, we add the buoyant force $B$ to vertical component of momentum equation.

$B=g\left[\frac{T-T_{0}}{T_{0}}+\epsilon\left(Y_{\mathrm{H}_{2} \mathrm{O}}-q_{0}\right)-q_{c}\right]$,

where $g=9.81 \mathrm{~m} \mathrm{~s}^{-2}$ is the acceleration of gravity, $q_{0}$ is the reference water vapour mixing ratio, $\epsilon+1=R_{\mathrm{v}} / R_{\mathrm{d}}$ is the ratio of the gas constant for water vapour and dry air, and $q_{c}$ is the cloud water mixing ratio (Andrejczuk et al., 2004; Babkovskaia et al., 2015). Note that the buoyancy force is applied to the air vertical acceleration/deceleration. The air temperature can evolve due to thermal diffusion, viscous heating, adiabatic contraction/expansion and latent heating/cooling caused by condensation/evaporation on the droplet surface. Coefficient of thermal conductivity and kinematic viscosity are calculated for a mixture of three air species.

We consider the evolution of aerosol number density function because of evaporation/condensation of aerosol particles. There is energy exchange between particles and ambient gas due to the release/absorption of latent heat caused by condensation/evaporation on the droplet surface. The motion of the particles is determined exclusively by their involvement in the motion of surroundings. In the present study we modify water vapour pressure over a droplet of radius $r$ as follows (Seinfeld and Pandis, 2006; Sorjamaa and Laaksonen, 2007):

$p_{\mathrm{vs}}=p_{0} \exp \left(\frac{A}{2 r}-\frac{0.1 d_{\mathrm{w}}}{2\left(r-r_{0}\right)}\right)$,

where $p_{0}$ is water vapour pressure over a flat surface at the same temperature, $A=0.66 / T$ (in $\mu \mathrm{m}$ ), $r_{0}=10 \mathrm{~nm}$ is the radius of the droplet core, $d_{\mathrm{w}}=0.3 \mathrm{~nm}$ is the size of water molecule.

For generating the initial turbulence, the external forcing $f$ is used in the following form:

$f(\boldsymbol{x}, t)=\operatorname{Re}\left\{N f_{k}(t) \exp [i \boldsymbol{k}(t) \cdot \boldsymbol{x}+i \phi(t)]\right\}$,

where $\boldsymbol{x}$ is the position vector. The wave vector $\boldsymbol{k}(t)$ and the random phase $-\pi<\phi(t) \leq \pi$ change at every time step; $N=f_{0} c_{\mathrm{S}}\left(|k| c_{\mathrm{s}} / \Delta t_{\mathrm{c}}\right)^{1 / 2}$ is the normalization factor, $c_{\mathrm{S}}$ is the sound speed, $f_{0}$ is a non-dimensional forcing amplitude; $\left.f_{k}=(\boldsymbol{k} \times \boldsymbol{e}) / \sqrt{\boldsymbol{k}^{2}-(\boldsymbol{k} \cdot \boldsymbol{e})^{2}}\right)$, where $\boldsymbol{e}$ is an arbitrary unit vector that is real and not aligned with $\boldsymbol{k}$ (see detail in Pencil Code, 2001).

To study the effect of aerosol on the turbulence we consider two initial turbulent fields, taking different nondimensional forcing amplitudes as $f_{0}=10$ (low intensive turbulence) and $f_{0}=100$ (high intensive turbulence). Also, we compare the effect of the aerosol in the case when the domain is in equilibrium with environment (equilibrium case) and when the temperatures inside and outside the domain are different (non-equilibrium case), taking the environment temperature $T_{0}=285.4 \mathrm{~K}$ for equilibrium case and $T_{0}=283.5 \mathrm{~K}$ for non-equilibrium case. The summary of four considered cases is in Table 2. To study the effect of total number of particles on activation, air temperature and supersaturation we consider the non-equilibrium case with low intensive turbulence.

\section{Results}

\subsection{Effect of total number of particles on air temperature and supersaturation distributions}

In Fig. 2 we present the air temperature averaged in $y$ direction when the aerosol dynamic is included. We find that in case 3 (see Fig. 2c) after $3 \mathrm{~s}$ the difference between the absolute value of maximal and minimal temperatures in the domain is about $0.02 \mathrm{~K}$, whereas without aerosol (case 5, not shown) after $3 \mathrm{~s}$ the temperature difference is still about $0.001 \mathrm{~K}$. Moreover, the temperature increases by about $1 \mathrm{~K}$ everywhere in the domain because of condensation of water vapour on aerosol particles.

Changing temperature distribution with time is mostly attributable to the periodic boundary conditions: the coldest layers are moving from the bottom to the middle of the domain. However, comparing panels a, b and c in Figs. 2 and 3 , we note that the positions of the coldest layers in different panels are different. The coldest layers in the case of $N_{\text {tot }}=55 \mathrm{~cm}^{-3}$ coincide with the layers where supersaturation is minimal (see panel a), whereas for other two cases, $N_{\text {tot }}=555 \mathrm{~cm}^{-3}$ (panel b) and $5550 \mathrm{~cm}^{-3}$ (panel c), the coldest layers correspond to the positions where supersaturation achieves maximum. It happens because of the different effect of aerosol dynamics in dependence on $N_{\text {tot }}$. The 

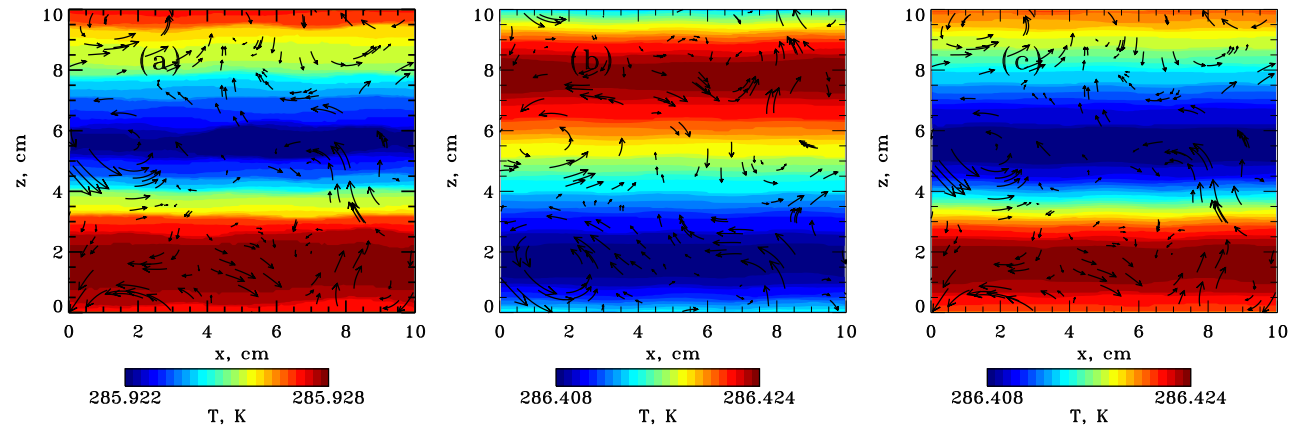

Figure 2. Temperature distribution at $t=3 \mathrm{~s}$ in case 1 (a), case 2 (b) and case 3 (c) (see Table 1). Non-equilibrium case with low intensive turbulence is considered (see Table 2). Velocity vector is shown by arrows (averaged vertical velocity is subtracted).
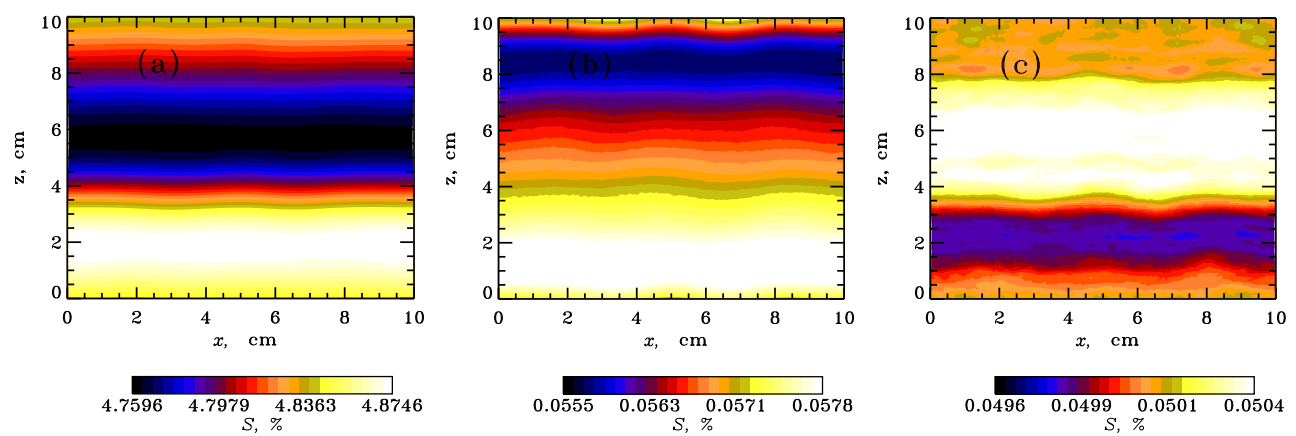

Figure 3. Distribution of supersaturation at $t=3 \mathrm{~s}$ in case 1 (a), case 2 (b) and case 3 (c), (see Table 1). Non-equilibrium case with low intensive turbulence is considered (see Table 2).

small amount of aerosol particles (case 1) does not make any substantial effect on the temperature distribution; i.e. both temperature and supersaturation are identically shifting with time in vertical direction. In turn, in cases 2 and 3 aerosol dynamics crucially change the temperature distribution. One can see in Figs. 2, 3 that layers with higher temperature correspond to layers with smaller supersaturation. It can be interpreted as follows: more intensive condensation occurs in initially warmer layers because supersaturation is larger there and the temperature increases faster in these layers. In turn, since supersaturation exponentially depends on temperature, $S \propto \exp (-T)$, at some moment $S$ appears to be smaller in warmer than cooler layers. Thus, equilibrium supersaturation is higher in the layers with temperature minimum (and vice versa). Also, we find that in case 1 at $t=3 \mathrm{~s}$ the supersaturation is about $5 \%$ and the aerosol is still activating, whereas in case 3 for the first $3 \mathrm{~s}$ the supersaturation almost drops to zero and the system is coming to an equilibrium.

Additionally, we investigate how fast the system with an initially high value of supersaturation $(S=10 \%)$ comes to an equilibrium $(S \simeq 0 \%)$ in dependence on different total numbers of droplets to answer the question how $N_{\text {tot }}$ affects the phase relaxation time. Analysing Fig. 3 we find that in a case of $N_{\text {tot }}=55 \mathrm{~cm}^{-3}$ it takes more than $3 \mathrm{~s}$ for the system to come to equilibrium; i.e. phase relaxation time appears to be larger than theoretically estimated turbulent mixing time $\left(\tau_{\text {eddy }}=2 \mathrm{~s}\right)$ (see discussion in Sect. 2$)$. Thus, strong supersaturation fluctuations can survive longer if the total number of droplets is small.

\subsection{Effect of total number of particles on activation}

In this study we consider that all particles with radii larger than $r_{\mathrm{cr}}=1.75 \mu \mathrm{m}$ are activated. This value is somewhat arbitrary, but the results of our study were not sensitive to the choice of $r_{\mathrm{cr}}$, provided that $r_{\mathrm{cr}} \leq 1.75 \mu \mathrm{m}$. In Fig. 1 we present the normalized particle distribution at $t=0 \mathrm{~s}$ and $t=3 \mathrm{~s}$. We find that the smaller the total number, the larger the particles produced for the similar time period. Comparing the supersaturation at $t=3 \mathrm{~s}$ in these three cases (Fig. 3), one can see that in the case of the largest $N_{\text {tot }}$ the supersaturation appears to be close to zero and particles stop growing, while for the smaller $N_{\text {tot }}$ the supersaturation is about $4.8 \%$ and particles continue growing. Therefore, the system comes to an equilibrium (i.e. $S \simeq 0$ ) faster and aerosol particles cease to grow earlier for the larger $N_{\text {tot }}$. Indeed, the phase relaxation time can be estimated as follows:

$\tau_{\text {phase }}=\left(a_{2} N_{\mathrm{tot}} \bar{r}\right)^{-1}$, 


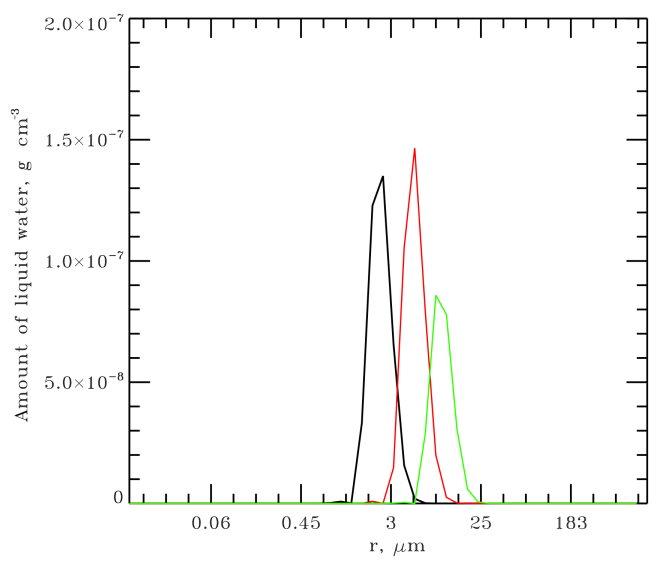

Figure 4. Amount of liquid water accumulated in particles with corresponding radius $r$ at $t=3 \mathrm{~s}$ (solid curve) for the cases of $N_{\text {tot }}=55.5 \mathrm{~cm}^{-3}$ (green solid curve), $N_{\text {tot }}=555 \mathrm{~cm}^{-3}$ (red solid curve) and $N_{\text {tot }}=5550 \mathrm{~cm}^{-3}$ (black solid curve).

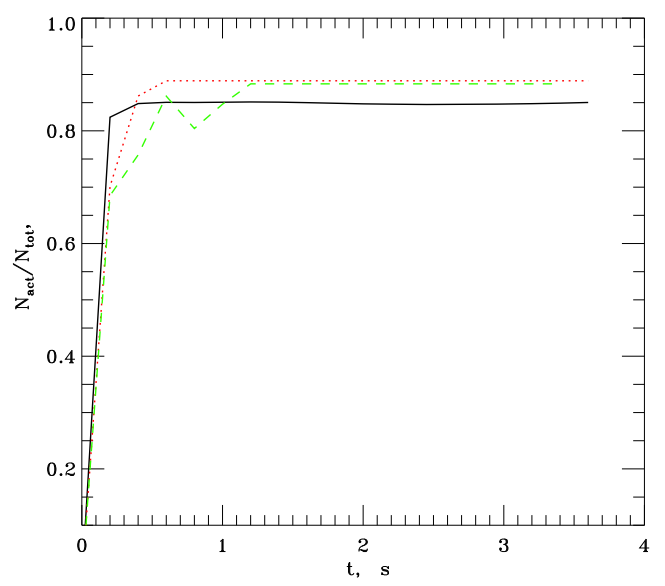

Figure 5. Relative number of activated particles as a function of time $N_{\text {tot }}=55.5 \mathrm{~cm}^{-3}$ (green curve), $N_{\text {tot }}=555 \mathrm{~cm}^{-3}$ (red curve) and $N_{\text {tot }}=5550 \mathrm{~cm}^{-3}$ (black curve).

where $\bar{r}$ is the mean droplet radius and $a_{2}=3.04 \times$ $10^{-4} \mathrm{~m}^{2} \mathrm{~s}^{-1}$. The steady-state supersaturation can be written as $S_{q s} \propto a_{1} w \tau_{\text {phase }}$, where $a_{1}$ is a parameter including thermodynamic parameters and being almost constant, $w$ is vertical velocity. Thus, it becomes clear that for larger $N_{\text {tot }}$ the system comes to an equilibrium faster. We show that aerosol particles can grow and possibly achieve the size of the rain drop only in the case of small total number of particles. This is consistent with the fact that in natural clouds, deep ascent is needed to drive the prolonged condensational growth of drops in order to form rain (e.g. Yau and Rogers, 1989, a short course in cloud physics). In real clouds, the ascent is the source of larger-scale supersaturation. One should also mention that in the scope of this model we neglect collisions and coalescence of aerosol particles (crucial in creation of rain drops) because of the short total simulation time. Also,

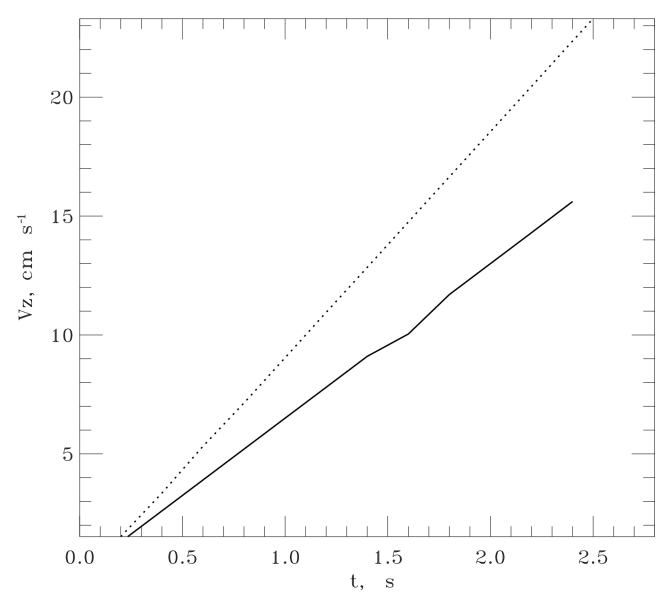

Figure 6. Averaged vertical velocity as a function of time with aerosol for $N_{\text {tot }}=5500 \mathrm{~cm}^{-3}$ (dotted curve, case 3) and without aerosol (solid curve, case 5). Non-equilibrium case with low intensive turbulence is considered (see Table 3 ).

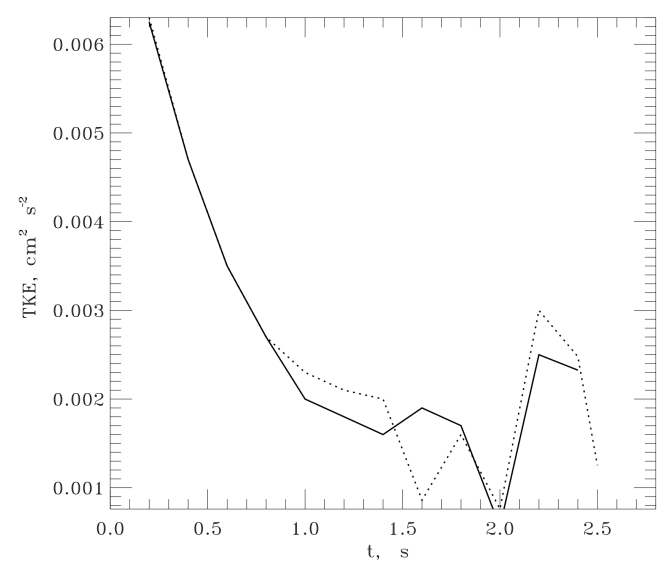

Figure 7. The dependence of the average turbulent kinetic energy on time with aerosol for $N_{\text {tot }}=5500 \mathrm{~cm}^{-3}$ (dotted curve, case 3 ) and without aerosol (solid curve, case 5). The other parameters correspond to non-equilibrium case with low intensive turbulence (see Table 2).

due to the short simulation time the droplets are too small for an effective coalescence process.

In addition, in Fig. 4 we analyse the amount of liquid water, $\operatorname{LW}(r)=(4 / 3) \pi r^{3} \rho_{\mathrm{w}} N(r)$, where $N(r)$ is the number of particles with radius $r$, and $\rho_{\mathrm{w}}$ is liquid water density. In Fig. 5 we show the fraction of activated particles averaged over the domain as a function of time in cases 1 , 2 and 3. In Table 3 we collect the most important quantities, such as initial value of supersaturation $\left(\overline{S_{\text {init }}}\right)$, number of activated particles $\left(N_{\text {act }}\right)$ at $t=3 \mathrm{~s}$, liquid water content $\mathrm{LWC}=\int \mathrm{LW}(r) / \Delta r \mathrm{~d} r$ (where $\Delta r$ is a size of corresponding bin), change in temperature between start and end of simulation $(\Delta T)$, change in percentage maximal supersaturation between start and end of simulation $\left(\Delta S_{\max }\right)$, change 


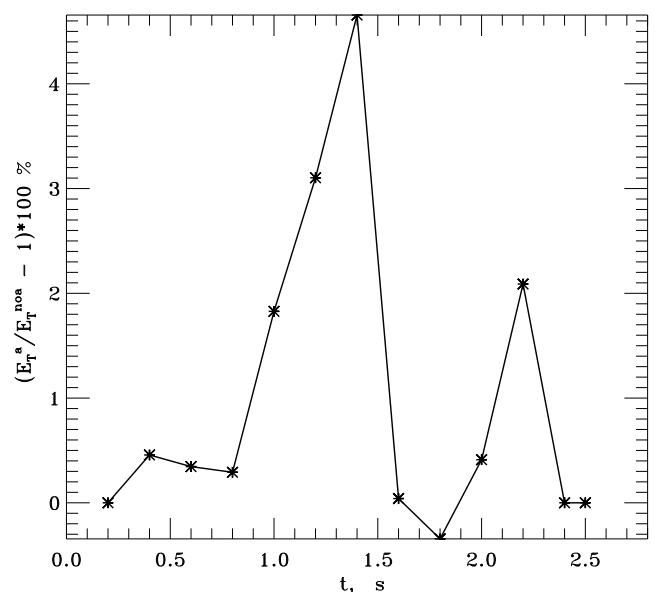

Figure 8. The difference between turbulent kinetic energy averaged over time period $t$ in a case without aerosol $\left(E_{T}^{\text {noa }}\right)$ and with aerosols $\left(E_{T}^{\mathrm{a}}\right)$, where $E_{T}(t)=1 / t \int_{0}^{t} \mathrm{TKE}\left(t^{\prime}\right) \mathrm{d} t^{\prime}$. All other parameters are the same as in Fig. 7.

Table 3. Initial value of supersaturation averaged over the domain $\overline{S_{\text {init }}}$, total number of particles $\left(N_{\text {tot }}\right)$, number of activated particles $\left(N_{\text {act }}\right)$ at $t=3 \mathrm{~s}$, liquid water content (LWC) at $t=3 \mathrm{~s}$, change in temperature between start and end of simulation $(\Delta T)$, change in percentage maximal supersaturation between start and end of simulation $\left(\Delta S_{\max }\right)$, change in percentage minimal supersaturation between start and end of simulation $\left(\Delta S_{\min }\right)$, the phase relaxation time of supersaturation $\tau_{\text {phase }}$ at $t=3 \mathrm{~s}$ for considered cases $1,2,3$ and 4 (see Table 1). The phase relaxation time $\tau_{\text {phase }}$ is the numerically predicted value in cases 2, 3 and 4 . In case 1 the phase relaxation time is obtained from Eq. (6). No subsaturation is predicted anywhere in the domain in all cases. Non-equilibrium case with low intensive turbulence is considered (see Table 2).

\begin{tabular}{lrrrr}
\hline & Case 1 & Case 2 & Case 3 & Case 4 \\
\hline$\overline{S_{\text {init }}}, \%$ & 10.3 & 10.3 & 10.3 & 0.6 \\
$N_{\text {tot }}, \mathrm{cm}^{-3}$ & 55.5 & 555 & 5550 & 5550 \\
$N_{\text {act }}, \mathrm{cm}^{-3}$ & 49 & 493 & 4700 & 395 \\
$\mathrm{LWC}_{\mathrm{g} \mathrm{m}} \mathrm{g} \mathrm{m}^{-3}$ & 0.23 & 0.369 & 0.376 & 0.0377 \\
$\Delta T, \mathrm{~K}$ & 0.52 & 1.0 & 1.0 & 0.05 \\
$\Delta S_{\max }, \%$ & -5.42 & -10.11 & -10.47 & -0.69 \\
$\Delta S_{\min }, \%$ & -5.66 & -10.12 & -10.48 & -0.37 \\
$\tau_{\text {phase }}, \mathrm{s}$ & 4 & 0.77 & 0.17 & 0.6 \\
\hline
\end{tabular}

in percentage minimal supersaturation between start and end of simulation $\left(\Delta S_{\min }\right)$, the phase relaxation time of supersaturation $\tau_{\text {phase }}$ for cases 1, 2, 3 and 4 (see Table 1). Note that no subsaturation is predicted anywhere in the domain in all considered cases.

We find that while the total number in case 2 is 10 times smaller than in case 3 , liquid water content is similar in these two cases. On the other hand, LWC appears to be smaller in case 1 than in cases 2, 3 (see Table 3 and Fig. 4). It happens because the probability of the water molecules catching

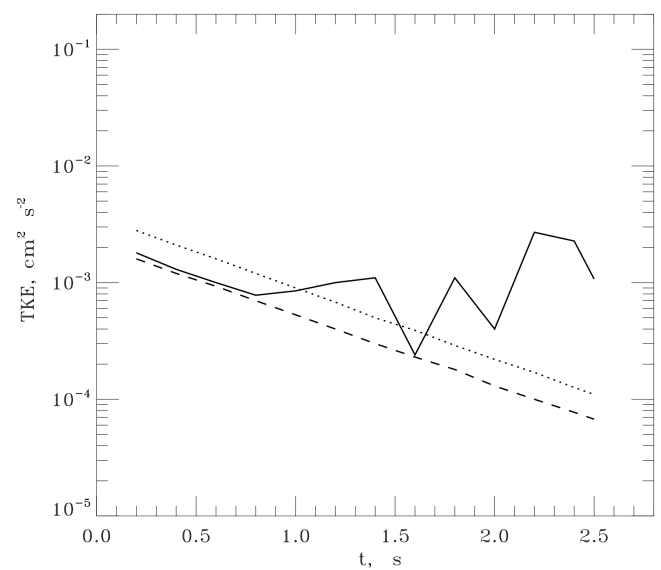

Figure 9. The dependence of the $x$ component (dotted curve), $y$ component (dashed curve) and $z$ component (solid curve) of the averaged kinetic energy on time with aerosol for $N_{\text {tot }}=5500 \mathrm{~cm}^{-3}$.

a particle is much smaller in case of the smallest particle concentration (case 1) than in cases 2, 3. In Fig. 5 one can see that in case 1 and case 2 the number of activated particles does not grow after 1.2 and $0.6 \mathrm{~s}$, correspondingly, and in case 3 it happens after $0.5 \mathrm{~s}$. Since in case 3 the equilibrium is achieved earlier than in cases 1 and 2, the maximum of final particle distribution in case 3 is shifted (see Fig. 1) and the final relative number of activated particles appears to be smaller than in cases 1 and 2 . We find that at $t=3 \mathrm{~s}$ the number of activated particles is proportional to the total number, whereas the change of $N_{\text {tot }}$ by a factor of a hundred increases LWC by approximately $40 \%$ (Table 3 ).

\subsection{Effect of aerosol on the turbulent motion}

We analyse the effect of aerosol on the turbulent motion, taking $N_{\text {tot }}=5500 \mathrm{~cm}^{-3}$ (case 3 in Table 1). First, we consider the equilibrium case, taking $T_{0}=285.4 \mathrm{~K}$ and $q_{0}=0.0124$ in Eq. (1). In that case the turbulent field appears to be isotropic. Next, we decrease $T_{0}=283.5 \mathrm{~K}$ and study the development of turbulence in the non-equilibrium case. In that case the intensive vertical motion is generated due to buoyant force. Note that the model domain is not vertically displaced during the simulation time, but the vertical motion is generated within the domain. Also, we vary parameter $f_{0}$ in Eq. (3) to compare the development of low intensive $\left(f_{0}=10\right)$ and high intensive $\left(f_{0}=100\right)$ turbulence in both equilibrium and non-equilibrium cases. The summary of parameters is presented in Table 2. Finally, we compare the results of simulations with and without particles in all four cases described above.

We find that the vertical motion of air is accelerated because of aerosol dynamics. Also, in Fig. 8 we show the time-averaged turbulent kinetic energy, $E_{T}(t)=$ $1 / t \int_{0}^{t} \operatorname{TKE}\left(t^{\prime}\right) \mathrm{d} t^{\prime}$, as a function of time in cases with aerosol particles and without them. We conclude that turbulent ki- 


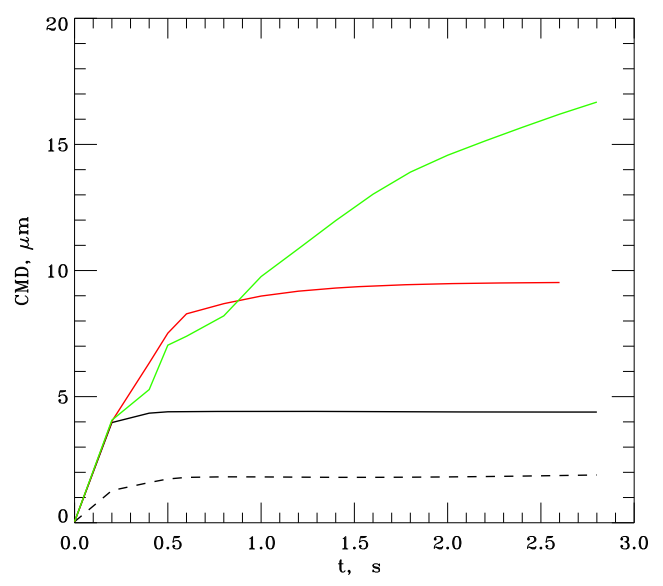

Figure 10. The dependence of count mean diameter (CMD) on time for case 1 (green solid curve), case 2 (red solid curve), case 3 (black solid curve), case 4 (black dashed curve).

netic energy increases because of the presence of aerosols. We interpret these results as follows: the air temperature increases because of the release of latent heat caused by condensation onto droplets, and therefore the difference between temperatures inside and outside the domain is enlarged. It results in the increase of buoyant force and acceleration of air motion in a vertical direction.

Also, we find that acceleration does not depend on intensity of turbulent fluctuations; i.e. acceleration in vertical direction is the same in low intensive turbulence and in high intensive turbulence cases. Moreover, turbulent fluctuations grow because of presence of aerosol particles in all four considered cases. The dependences of vertical velocity and turbulent kinetic energy (TKE) averaged over the domain as a function of time for non-equilibrium case with low intensive turbulence are presented in Figs. 6 and 7, correspondingly.

Finally, we find that there is a strong variation of TKE with time (see Fig. 7). To interpret this fact we plot $x, y$ and $z$ components of TKE in Fig. 9 and find strong time variations only in the $z$ component of TKE ( $x$ and $y$ components are smoothly decreasing with time). We conclude that it results from fluctuations of temperature caused by aerosol dynamics, and therefore changes of buoyant force with time, which result in perturbation of vertical motion.

\subsection{Effect of initial supersaturation on activation of aerosol particles}

To illustrate the effect of initial supersaturation on activation of aerosol particles we modify the initial distribution of absolute humidity in case 3 , decreasing it by $10 \%$. In that case the initial supersaturation averaged over the domain becomes $0.6 \%$. To study the effect of initial supersaturation on the activation of aerosol particles in Fig. 10, we analyse count me-

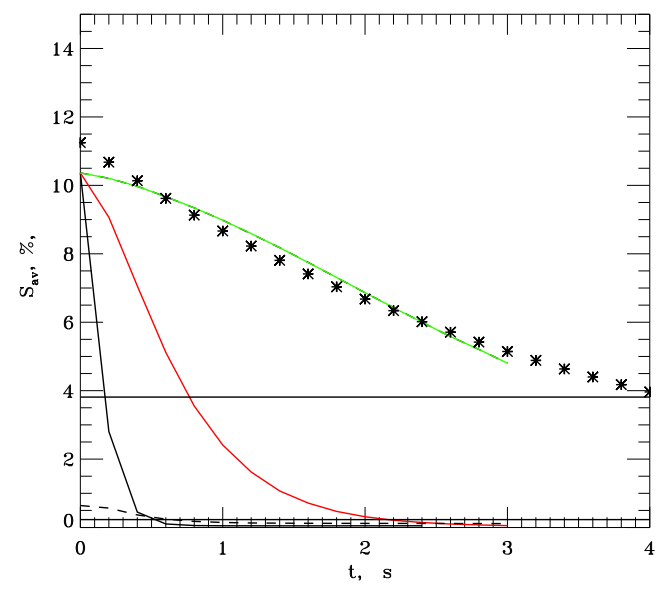

Figure 11. The dependence of supersaturation averaged over the calculation domain on time for case 1 (green solid curve), case 2 (red solid curve), case 3 (black solid curve), case 4 (black dashed curve). Horizontal lines show the corresponding values of $\overline{S_{\text {init }}} / e$. Asterisks show the extrapolation function in case 1 (see Eq. 6).

dian diameter (CMD).

$\mathrm{CMD}=\left(D_{1}^{n_{1}} D_{2}^{n_{2}} D_{3}^{n_{2}} \ldots D_{N_{\mathrm{tot}}}^{n_{N_{\mathrm{tot}}}}\right)^{1 / N_{\mathrm{tot}}}$,

where $D_{i}$ is particle size of the $i$ th bin and $n_{i}$ is number of particles, having a size $D_{i}$. Dependences of CMD on time in cases 1, 2, 3 and 4 are presented in Fig. 10. Comparing cases 3 and 4, we find that in both the system comes to equilibrium at $t \simeq 0.6 \mathrm{~s}$. In other words, the typical time it takes for droplets to cease growing does not depend on initial supersaturation. On the other hand, a value of CMD in equilibrium crucially depends on supersaturation and it appears to be 2 times larger in case 3 than in case 4 .

Additionally, in Fig. 11 we present the supersaturation averaged over domain for cases 1, 2, 3 and 4 (see Table 1) and analyse the phase relaxation time of supersaturation $\left(\tau_{\text {phase }}\right)$ for different values of initial supersaturation and total number of particles. From analysing numerical results, we get the phase relaxation time of about $0.77 \mathrm{~s}$ (case 2), $0.17 \mathrm{~s}$ (case 3) and $0.6 \mathrm{~s}$ (case 4). Using Eq. (4) and count median diameter (see Fig. 10), we estimate the phase relaxation time of $1.23 \mathrm{~s}$ (case 2), $0.27 \mathrm{~s}$ (case 3) and $0.63 \mathrm{~s}$ (case 4). Therefore, we conclude that results of our simulations are in a good agreement with analytical estimations. In case 1 the phase relaxation time is larger than the simulation time. In that case we extrapolate the numerical results with the following function (see asterisk in Fig. 11):

$S_{\mathrm{av}}(t)=11.25 \exp \left(-\frac{t}{3.84}\right)$,

and estimate the phase relaxation time of about $4 \mathrm{~s}$. The results are summarized in Table 3 . We conclude that $\tau_{\text {phase }}$ depends both on initial supersaturation and on the total number of particles. 


\section{Summary}

Turbulence, aerosol growth and microphysics of hydrometeors in clouds are intimately coupled. In the present study a new modelling approach was applied so as to quantify this link. We study the interaction in the cloud area under transient, high supersaturation conditions, using direct numerical simulations. As the initial conditions, we take observational data. To analyse the effect of aerosol and droplets on turbulence, a small volume with supersaturation of $10 \%$ was considered. Under such extreme conditions, condensation is the dominant process. The results cannot be linearly extended to bigger cloud volumes but should be considered relevant for a small cloud parcel with extreme supersaturation due to turbulent mixing of the water vapour and temperature field. As an initial distribution of particles, we take the data of measurements at the sea level and analyse the droplets activated by aerosols in the simulations.

To study the effect of total number of particles on activation, air temperature and supersaturation, we vary the total number of particles and take the other parameters which correspond to low intensive turbulence and non-equilibrium case, i.e. when the vertical motion is generated within domain because of buoyancy. We compare the results of simulations with particles and without them. We find that the total number of particles in the domain is crucial for the distribution of temperature and for developing turbulence. Even small amounts of aerosol particles $\left(55.5 \mathrm{~cm}^{-3}\right)$, and therefore small cloud droplet number concentrations, increase the air temperature by $1 \mathrm{~K}$ because of latent heating caused by condensation onto drops. The system comes to an equilibrium faster with a larger total number of particles. To illustrate the effect of initial supersaturation on activation of aerosol particles, we compare the results of simulations with initial supersaturation averaged over a domain of 10.3 and $0.6 \%$. We conclude that the typical time it takes for droplets to cease growing does not depend on initial supersaturation. Also, we analyse the phase relaxation time of supersaturation (i.e. time of dropping from initial value to $1 / e=0.368$ ) for different values of initial supersaturation and total number of particles. We find that the phase relaxation time crucially depends both on the total number of particles and on initial supersaturation.

To analyse the effect of aerosol dynamics on the turbulent kinetic energy and on vertical velocity, we take the maximal value of $N_{\text {tot }}=5550 \mathrm{~cm}^{-3}$. We conclude that the presence of aerosol has an effect on vertical motion and, in our case (when the temperature inside the domain is larger than in environment), tends to enlarge upward velocity. We conclude that aerosols quite strongly influence the dynamics in the cloud area. Such effect of aerosols can also be crucial on large scales, usually studied with large eddy simulation (LES), and the LES parameterization can be improved with direct numerical simulations.

\section{Data availability}

The description of data from CARRIBA project has been published in Siebert et al. (2013).

Acknowledgements. We thank the Helsinki University Centre for Environment (HENVI) and computational resources from CSC - IT Center for Science Ltd are all gratefully acknowledged. This research is supported by the Academy of Finland Center of Excellence program (project number 272041). Anthropogenic emissions on Clouds and Climate: towards a Holistic UnderStanding (BACCHUS), project no. 603445.

Edited by: S. M. Noe

\section{References}

Andrejczuk, M., Grabowski, W., Malinowski, S., and Smolarkiewicz, P.: Numerical simulations of Cloud-clear air interfacial mixing, J. Atmos. Sci., 61, 1726-1739, 2004.

Babkovskaia, N., Haugen, N., and Brandenburg, A.: A high-order public domain code for direct numerical simulations of turbulent combustion, J. Comput. Phys., 230, 1-12, 2011.

Babkovskaia, N., Boy, M., Smolander, S., Romakkaniemi, S., Rannik, U., and Kulmala, M.: A study of aerosol activation at the cloud edge with high resolution numerical simulations, Atmos. Res., 153, 49-58, 2015.

Benmoshe, N. and Khain, A. P.: The effects of turbulence on the microphysics of mixed-phase deep convectiveclouds investigated with a $2-D$ cloud model with spectral bin microphysics, J. Geophys. Res.-Atmos., 119, 207-221, 2014.

Ditas, F., Shaw, R. A., Siebert, H., Simmel, M., Wehner, B., and Wiedensohler, A.: Aerosols-cloud microphysicsthermodynamics-turbulence: evaluating supersaturation in a marine stratocumulus cloud, Atmos. Chem. Phys., 12, 2459-2468, doi:10.5194/acp-12-2459-2012, 2012.

Dobler, W., Stix, M., and Brandenburg, A.: Magnetic field generation in fully convective rotating spheres, Astrophys. J., 638, 336343, 2006.

Easter, R. C. and Peters, L. K.: Binary homogeneous nucleation: Temperature and relative humidity fluctuations, non-linearity and aspects of new particle production in the atmosphere, J. Appl. Meteorol., 33, 775-784, 1994.

Forster, P.: Changes in atmospheric constituents and in radiative forcing Climate Change 2007: The Physical Science Basis, edited by: Solomon, S., Qin, D., Manning, M., Chen, Z., Marquis, M., Averyt, K. B., Tignor, M., and Miller, H. L., Cambridge University Press, 129-234, 2007.

Katzwinkel, J., Siebert, H., Heus, T., and Shaw, R. A.: Measurements of turbulent mixing and subsiding shells in trade wind cumuli, J. Aerosol Sci., 71, 2810-2822, 2014.

Korolev, A. V. and Mazin, I. P.: Supersaturation of Water Vapor in Clouds, J. Atmos Sci., 60, 2957-2974, 2003.

Kulmala, M., Rannik, U., Zapadinski, E., and Clement, C. F.: The effect of saturation fluctuations on droplet growth, J. Aerosol Sci., 28, 1395-1409, 1997. 
Nilsson, E. D. and Kulmala, M.: The potential for atmospheric mixing processes to enhance the binary nucleation rate, J. Geophys. Res., 103, 1381-1389, 1998.

Nilsson, E. D., Pirjola, L., and Kulmala, M.: The effect of atmospheric waves on aerosol nucleation and size distribution, J. Geophys. Res., 105, 19917-19926, 2000.

The PENCIL Code: available at: http://pencil-code.nordita.org/doc/ manual.pdf (last access: 23 June 2016), 2001.

Seinfeld, J. H. and Pandis, S. N.: Atmospheric chemistry and physics: From Air pollution to Climate Change, John Wiley \& Sons, Inc., Cambridge University Press, Cambridge, United Kingdom and New York, NY, USA, 2006.

Shaw, R. A.: Supersaturation intermittency in turbulent clouds, J. Atmos. Sci., 57, 3452-3456, 2000.

Siebert, H., Lehmann, K., and Wendisch, M.: Observations of small scale turbulence and energy dissipation rates in the cloudy boundary layer, J. Atmos. Sci., 63, 1451-1466, 2006.

Siebert, H., Shaw, R. A., and Warhaft, Z.: Statistics of Small-Scale Velocity Fluctuations and Internal Intermittency in Marine Stratocumulus Clouds, J. Atmos. Sci., 67, 262-273, 2010.
Siebert, H., Beals, M., Bethke, J., Bierwirth, E., Conrath, T., Dieckmann, K., Ditas, F., Ehrlich, A., Farrell, D., Hartmann, S., Izaguirre, M. A., Katzwinkel, J., Nuijens, L., Roberts, G., Schäfer, M., Shaw, R. A., Schmeissner, T., Serikov, I., Stevens, B., Stratmann, F., Wehner, B., Wendisch, M., Werner, F., and Wex, H.: The fine-scale structure of the trade wind cumuli over Barbados - an introduction to the CARRIBA project, Atmos. Chem. Phys., 13, 10061-10077, doi:10.5194/acp-13-10061-2013, 2013.

Sorjamaa, R. and Laaksonen, A.: The effect of $\mathrm{H}_{2} \mathrm{O}$ adsorption on cloud drop activation of insoluble particles: a theoretical framework, Atmos. Chem. Phys., 7, 6175-6180, doi:10.5194/acp-76175-2007, 2007.

Yau, M. K. and Rogers, R. R.: A Short Course in Cloud Physics, 3rd Edn. (International Series in Natural Philosophy), ButterworthHeinemann, 1989. 